Original paper

\title{
Liver function test abnormalities in COVID-19 patients and factors affecting them - a retrospective study
} \author{
Noha Saber Shafik ${ }^{4}$ \\ 'Tropical Medicine and Gastroenterology Department, Faculty of Medicine, Sohag University, Egypt \\ IInternal Medicine Department, Faculty of Medicine, Sohag University, Egypt \\ ${ }^{3}$ Clinical and Chemical Pathology Department, Faculty of Medicine, Sohag University, Egypt \\ ${ }^{4}$ Medical Microbiology and Immunology Department, Faculty of Medicine, Sohag University, Egypt
}

Mona Mohammed Abdelrahman', Asmaa A. Abdel-baset ${ }^{2}$, Mustafa Adel Younis ${ }^{3}$, Mahmoud Gaber Mahmoud²,

\begin{abstract}
Aim of the study: We aimed to study liver function test abnormalities in our COVID-19 patients and factors affecting them and to evaluate whether liver function test abnormalities are related to the severity of COVID-19.

Material and methods: Our retrospective study included 118 patients who were SARS-CoV-2 positive. Their median age was 40 years. Fifty percent were male. Clinical and biochemical data were collected from patient records during the period from the start of June 2020 to the end of July 2020. Liver function test abnormalities included: alanine aminotransferase (ALT) > $40 \mathrm{U} / \mathrm{l}$, aspartate aminotransferase (AST) $>40 \mathrm{U} / \mathrm{l}$, serum albumin $<3.5 \mathrm{mg} / \mathrm{dl}$, total bilirubin $>1.2 \mathrm{mg} / \mathrm{dl}$, and international normalized ratio (INR) $>1.2$.

Results: Forty-four percent of COVID-19 patients had liver function test (LFT) abnormalities. In patients with severe SARS-CoV-2, AST, total bilirubin and INR levels were significantly higher than in patients with the nonsevere disease. Levels of hemoglobin, C-reactive protein (CRP), erythrocyte sedimentation rate (ESR), serum ferritin, D-dimer, and serum glucose were significantly higher in SARS-CoV-2 patients with LFT abnormalities than those with normal liver function.
\end{abstract}

Conclusions: LFT abnormalities are very common in SARS-CoV2 positive patients, especially those with the severe form. Levels of ESR, CRP, serum ferritin, and D-dimer were higher in COVID-19 patients with LFT abnormalities than those with normal LFT. High serum ferritin levels might be potential risk factors for LFT abnormalities.

Key words: liver function tests, COVID-19, risk factors.

Address for correspondence:

Dr. Mona Mohammed Abdelrahman, Tropical Medicine and Gastroenterology Department, Faculty of Medicine, Sohag University, Egypt, e-mail: Monamohamed@med.sohag.edu.eg

\section{Introduction}

Coronaviruses that cause human infection were first discovered in the mid-1960s. Coronaviruses are positive-strand-enveloped RNA viruses. Most of them cause mild symptoms, with two exceptions: severe acute respiratory distress coronaviruses (SARS-CoV-1) and Middle East respiratory syndrome coronavirus (MERS-CoV) [1]. By the end of 2019, a large pandemic due to the emergence of severe acute respiratory syndrome coronavirus 2 (SARS-CoV-2) had started in China [2].
By 6 March 2021, over 115 million confirmed cases of COVID-19 had been recorded worldwide [3]. The most common symptoms of SARS-CoV-2 infection are fever, cough, and breathing difficulties [4]. Also, a variety of systematic symptoms including the gastrointestinal tract are reported [5].

With the growing number of patients infected with COVID-19, multiple studies have indicated that the liver is the organ most commonly involved after lung damage $[6,7]$. SARS-CoV-2 reaches the target cell by attaching to angiotensin-converting enzyme 2 (ACE2), which is extensively expressed 
on hepatocytes (2.6\%), cholangiocytes (59.7\%), and hepatic endothelial cells [6]. This may to some extent explain the cause of liver function test abnormalities in SARS-CoV-2 positive patients [7]. Also, the role of immune-mediated inflammation and underlying chronic liver disorder as a reason for liver function test abnormalities should be taken into consideration [8].

Hepatic insult has been often reported as a temporary increase of transaminases (alanine aminotransferase $[\mathrm{ALT}]$, aspartate aminotransferase $[\mathrm{AST}])$. However, acute liver injury has been detected less often [9]. So, in our study, we aimed to investigate liver function test (LFT) abnormalities in COVID-19 patients and factors affecting them and to evaluate whether liver function test abnormalities are related to the severity of COVID-19.

\section{Material and methods}

This retrospective study was carried out on $118 \mathrm{pa}$ tients ( 59 males, 59 females) who were diagnosed as SARS-CoV-2 positive. We retrospectively collected the data from patient records in COVID-19 isolation hospitals in Sohag governorate and the isolation department in Sohag university hospitals during the period from the start of June 2020 to the end of July 2020. The inclusion criteria were patients with positive PCR for SARS-CoV-2, while the exclusion criteria were patients with chronic hepatic disorders (liver cirrhosis, hepatitis B surface antigen-positive, or patients who were anti-HCV antibody positive and HCV RNA positive) and patients with a history of alcohol intake.

The study protocol was accepted by the Ethical Committee of Scientific Research, Faculty of Medicine, Sohag University. The researchers retrospectively collected data about clinical manifestations including fever, cough, dyspnea, fatigue, and diarrhea, and laboratory investigation: complete blood count (CBC) by $A B X$ Micros 60 (Horriba-ABX, France), total and direct bilirubin, AST, ALT, albumin, C-reactive protein (CRP) by Vitros-350 autoanalyzer (Ortho-Clinical Diagnostics, USA), prothrombin time and concentration by STA-Satellite (Diagnostic STAGO, France), D-dimer by Cobas h232 from Roche Diagnostic (Germany) and serum ferritin by TOSOH AIA-360 from Tosoh Corporation, Japan.

\section{SARS-CoV-2 RNA recognition using real-time polymerase chain reaction}

Nasal specimens were obtained by mid-turbinate swabbing of both nostrils. Oropharyngeal specimens were gathered by swabbing the posterior pharynx. For the processing of all clinical samples, flocked swabs were used and treated as recommended in international guidelines. The existence of SARS-CoV-2 RNA in the samples was determined based on a quantitative real-time polymerase chain reaction using a Daan novel kit from Da An Gene Co., Ltd. Of Sun Yat-sen University. DNA was extracted using the QIA symphony RGQ system from Qiagen. The presence of SARS-CoV-2 was detected by Rotor-Gene 6000 from Corbett Research, Australia. The primers were developed to identify the open reading frame $1 \mathrm{ab}(\mathrm{ORF} 1 \mathrm{ab})$ and the protein nucleocapsid $(\mathrm{N})$ to be multiplied and tested. The kit has an internal monitoring detection system with a detection process that runs in triplicate with positive and negative control sets, not to be considered invalid [7].

\section{Severity of SARS-CoV-2 infection}

Depending on findings from computed tomography (CT) of the chest, clinical evaluation, and complaints, the patients were categorized into severe and non-severe cases:

- patients with mild symptoms (fever, cough, and other complaints of respiratory tract system), and without changes, or with mild findings on CT (multiple small patchy shadows and interstitial shifts, especially in the outer zone of the lung and under the pleura) were categorized as non-severe types;

- the existence of one or more of the following criteria was identified as severe SARS-CoV-2 infection: i) respiratory rate $(\mathrm{RR}) \geq 30$ times/minute, ii) oxygen saturation at room air and resting condition $\leq 92 \%$, iii) arterial blood gas measurement: partial pressure of oxygen/fraction of inspired oxygen $\left(\mathrm{PaO}_{2} / \mathrm{FiO}_{2}\right)$ $\leq 300 \mathrm{mmHg}$, CT of the chest revealing more than $50 \%$ lesion or progressive lesion within 24 to $48 \mathrm{~h}$. Critically ill patients were diagnosed if $\mathrm{SaO}_{2}<92$, or $\mathrm{RR}>30$, or $\mathrm{PaO}_{2} / \mathrm{FiO}_{2}$ ratio $<200$ despite oxygen therapy [10].

\section{Liver function test abnormalities}

Liver function test abnormalities included: ALT more than $40 \mathrm{U} / \mathrm{l}$, AST more than $40 \mathrm{U} / \mathrm{l}$, serum albumin less than $3.5 \mathrm{mg} / \mathrm{dl}$, serum bilirubin more than $1.2 \mathrm{mg} / \mathrm{dl}$, international normalized ratio (INR) more than 1.2. Acute liver injury (ALI) was defined as an increase in the levels of ALT and/or AST three times more than the upper limit of normal.

\section{Statistical analysis}

SPSS version 16 was used to analyze the results. Continuous data were analyzed as mean \pm SD or me- 
dian and IQR, and categorical data were analyzed as numbers and percentages. The Student $t$-test or Mann-Whitney $U$ test for continuous data was used to compare differences between the two groups, while the $\chi^{2}$ test was used to compare categorical data. Logistic regression analysis was conducted to evaluate risk factors of LFT abnormalities.

\section{Results}

A total of 118 patients with COVID-19 infection who fulfilled the study's inclusion criteria were included in our study. Their median age was 40 years with an age range of 11-78 years. Fifty percent of the study population were male. Fever, headache, fatigue, sore throat, and cough were the most frequent presenting complaints of COVID-19 infected patients in our study, while diarrhea had been reported in 37 patients (31.4\%). We found that COVID-19 infected patients with LFT abnormalities were older than those with normal LFTs. The temperature and the number of diabetic patients were significantly higher in patients with LFT abnormalities than those with normal LFTs (Table 1).

In the current study, at the time of admission, we found that $44 \%$ (52 out of 118) of COVID-19 infected patients had LFT abnormalities. We found that $39.8 \%$ (47 out of 118) had elevated liver enzymes. Elevation of both ALT and AST was detected in $24.6 \%$ (29 out of 118) patients. The percentages of ALT and AST elevation were equal (32\%). The authors also found that

Table 1. Characteristics of 118 SARS-CoV-2 positive patients at the time of admission according to liver function test abnormalities

\begin{tabular}{|c|c|c|c|c|}
\hline Variable & Total $(N=118), n(\%)$ & Normal $(n=66), n(\%)$ & Abnormal $(n=52), n(\%)$ & $P$-value \\
\hline Age (years), mean $\pm S D$ & $42 \pm 15$ & $40.03 \pm 14.2$ & $45.2 \pm 14.34$ & 0.06 \\
\hline$<20$ & $1(0.8)$ & $1(1.5)$ & $0(0)$ & \\
\hline $20-40$ & $59(50)$ & $38(57.57)$ & $21(40.38)$ & \\
\hline $41-60$ & $46(39)$ & $20(30.30)$ & $26(50)$ & \\
\hline $61-80$ & $12(10.2)$ & $7(10.6)$ & $5(9.6)$ & \\
\hline Gender & & & & 0.14 \\
\hline Male & $59(50)$ & $29(43.94)$ & $30(57.69)$ & \\
\hline Female & $59(50)$ & $37(56.06)$ & $22(42.31)$ & \\
\hline \multicolumn{5}{|l|}{ Comorbid conditions } \\
\hline DM & $23(19.5)$ & $6(9.09)$ & $17(32.69)$ & 0.001 \\
\hline Hypertension & $20(16.9)$ & $11(11.67)$ & $9(17.31)$ & 0.56 \\
\hline Chronic renal failure & $5(4.23)$ & $3(4.54)$ & $2(3.85)$ & 0.85 \\
\hline Coronary heart disease & $7(5.9)$ & $2(3.03)$ & $5(9.61)$ & 0.13 \\
\hline Chronic obstructive lung diseases & $5(4.23)$ & $1(1.51)$ & $4(7.69)$ & 0.098 \\
\hline \multicolumn{5}{|l|}{ Reported symptoms } \\
\hline Fever & $100(84.7)$ & $53(80.3)$ & $47(90.38)$ & 0.13 \\
\hline Cough & $59(50)$ & $39(59.1)$ & $29(55.77)$ & 0.7 \\
\hline Dyspnea & $51(43.2)$ & $24(36.36)$ & $27(51.92)$ & 0.09 \\
\hline Chest pain & $39(33.1)$ & $22(33.33)$ & $17(32.69)$ & 0.94 \\
\hline Sore throat & $65(55.1)$ & $37(56.06)$ & $28(53.85)$ & 0.81 \\
\hline Headache & $80(67.8)$ & $47(71.21)$ & $33(36.46)$ & 0.37 \\
\hline Fatigue & $82(69.5)$ & $43(65.15)$ & $39(75)$ & 0.25 \\
\hline Diarrhea & $37(31.4)$ & $23(34.48)$ & $14(26.92)$ & 0.35 \\
\hline Temperature $\left({ }^{\circ} \mathrm{C}\right)$, mean $\pm \mathrm{SD}$ & $37.95 \pm 1.2$ & $37.75 \pm 1.4$ & $38.2 \pm 0.76$ & 0.04 \\
\hline$<37.3$ & $18(15.3)$ & $14(21.2)$ & $4(7.7)$ & \\
\hline $37.3-38.0$ & $49(41.5)$ & $27(40.9)$ & $22(42.3)$ & \\
\hline $38.1-39.0$ & $42(35.6)$ & $23(34.8)$ & $19(36.5)$ & \\
\hline$>39$ & $9(7.6)$ & $2(3.03)$ & $7(13.5)$ & \\
\hline
\end{tabular}

$N$ - number, SD - standard deviation 


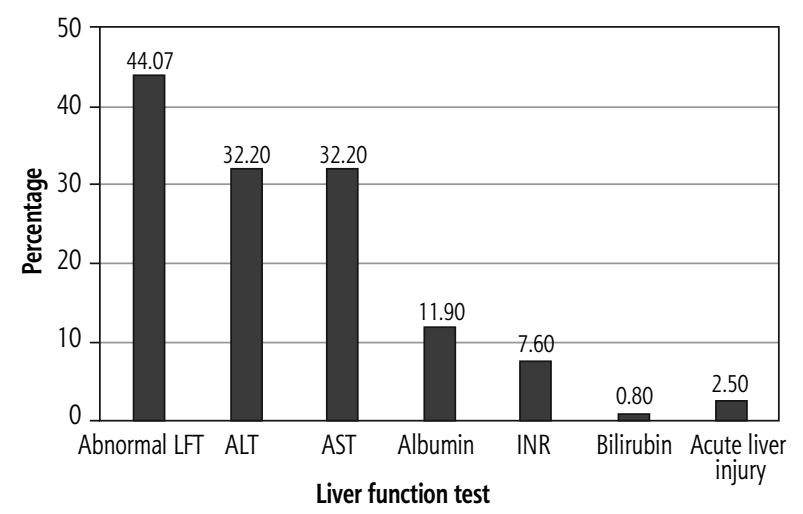

LFT - liver function test, ALT - alanine aminotransferase, AST - aspartate aminotransferase, INR - international normalized ratio

Fig. 1. Incidence of liver function test abnormalities in 118 SARS-CoV-2 positive patients

$11.8 \%$ (14 out of 118 ) of patients had a reduction in serum albumin less than the normal range $(3.5 \mathrm{mg} / \mathrm{dl})$ and $7.6 \%$ (9 out of 118) had elevated INR. Only one patient had elevated serum total bilirubin. Acute hepatic injury was detected in $2.5 \%$ ( 3 out of 118) of patients (Fig. 1).
The authors noted that LFT abnormalities including AST, total bilirubin, and INR were significantly higher in patients with severe COVID-19 than patients with the non-severe form. The elevations in transaminases (ALT, AST) are usually mild, with only three patients who had elevated transaminases more than 3 times the normal level. The highest value for AST and ALT was $127 \mathrm{IU} / 1,258 \mathrm{IU} / \mathrm{l}$, respectively. Also, serum albumin level was significantly lower in patients with severe disease. The reduction in serum albumin, elevation of bilirubin, and prolongation of INR were usually mild (Table 2).

In this study, we found that the levels of hemoglobin, CRP, erythrocyte sedimentation rate (ESR), serum ferritin, D-dimer, and serum glucose were significantly higher in SARS-CoV-2 patients with LFT abnormalities than those with normal LFTs (Table 3). We also conducted multivariate logistic regression to evaluate potential risk factors for LFT abnormalities. These variables included age, temperature, hemoglobin, CRP, ESR, D-dimer, ferritin, and glucose. We found that increased hemoglobin levels and high serum ferritin

Table 2. Incidence of liver function test abnormalities according to the severity of SARS-CoV-2

\begin{tabular}{|c|c|c|c|c|}
\hline Liver function tests & Total $(N=118), n(\%)$ & Non-severe $(n=93), n(\%)$ & Severe $(n=25), n(\%)$ & $P$-value \\
\hline $\operatorname{ALT}(\mathrm{U} / \mathrm{I})$, median (IQR) & $30.5(20-45.75)$ & $29(18-45)$ & $35(27-61)$ & 0.06 \\
\hline Normal & $80(67.8)$ & $64(68.82)$ & $16(64)$ & \\
\hline $1-2$ & $31(26.3)$ & $25(26.88)$ & $6(24)$ & \\
\hline $2-3$ & $4(3.4)$ & $2(2.15)$ & $2(8)$ & \\
\hline$>3$ & $3(2.5)$ & $2(2.15)$ & $1(4)$ & \\
\hline AST (U/I), median (IQR) & $32(21.7-44.25)$ & $29(20-44)$ & $38(31-45)$ & 0.04 \\
\hline Normal & $80(67.8)$ & $65(69.89)$ & $15(60)$ & \\
\hline $1-2$ & $30(24.4)$ & $22(23.65)$ & $8(32)$ & \\
\hline $2-3$ & $6(5.1)$ & $4(4.3)$ & $2(8)$ & \\
\hline$>3$ & $2(1.7)$ & $2(2.15)$ & $0(0)$ & \\
\hline Albumin (g/dl), median (IQR) & $3.95(3.78-4.33)$ & $4(3.8-4.4)$ & $3.6(3.4-3.75)$ & 0.000 \\
\hline Normal & $104(88.1)$ & $86(92.5)$ & $18(72)$ & \\
\hline $3.5-2.8$ & $14(11.9)$ & $7(7.5)$ & $7(28)$ & \\
\hline$<2.8$ & $0(0)$ & $0(0)$ & $0(0)$ & \\
\hline Total bilirubin (mg/dl), median (IQR) & $0.6(0.43-0.78)$ & $0.54(0.42-0.67)$ & $0.8(0.7-0.95)$ & 0.000 \\
\hline Normal & 117 (99.2) & $93(100)$ & $24(96)$ & \\
\hline $1-2$ & $1(0.8)$ & $0(0)$ & $1(4)$ & \\
\hline $2-3$ & $0(0)$ & $0(0)$ & $0(0)$ & \\
\hline INR, median (IQR) & $0.91(0.73-1)$ & 0.83 (0.7-0.99) & $1(1.1-1.3)$ & 0.000 \\
\hline Normal & $109(92.4)$ & $91(97.8)$ & $18(72)$ & \\
\hline $1-2$ & $8(6.8)$ & $2(2.2)$ & $6(24)$ & \\
\hline $2-3$ & $1(0.8)$ & $0(0)$ & $1(4)$ & \\
\hline$>3$ & $0(0)$ & $0(0)$ & $0(0)$ & \\
\hline
\end{tabular}

$N$ - number, IQR - interquartile range 
Table 3. Laboratory characteristics of 118 SARS-CoV-2 positive patients at admission by liver function tests

\begin{tabular}{|c|c|c|c|c|}
\hline Parameter & Total & $\begin{array}{l}\text { Patients with normal LFTs } \\
\qquad(n=66)\end{array}$ & $\begin{array}{c}\text { Patients with LFT } \\
\text { abnormalities }(n=52)\end{array}$ & $P$-value \\
\hline WBCs $\left(10^{9} / \mathrm{I}\right)$, median (IQR) & $6.8(4.58-8.92)$ & $6.95(4.35-9.1)$ & $6.7(5-8.9)$ & 0.73 \\
\hline Lymphocytes $\left(10^{9} / \mathrm{l}\right)$, median (IQR) & $1.2(1.55-2.52)$ & $1.47(1.25-2.39)$ & $1.8(1.03-2.7)$ & 0.56 \\
\hline Hemoglobin (g/dl), median (IQR) & $12.35(10.8-13.52)$ & $11.7(10.77-13.1)$ & $12.75(11-14.1)$ & 0.04 \\
\hline Platelets $\left(10^{9} / \mathrm{l}\right)$, median (IQR) & $230(188-280)$ & $239(191-308)$ & $216(180-259)$ & 0.12 \\
\hline $\operatorname{ALT}(\mathrm{U} / \mathrm{I})$, median (IQR) & $30.5(20-45.75)$ & $22.5(17-30.25)$ & $50.5(37-67)$ & 0.000 \\
\hline AST (U/I), median (IQR) & $32(21.75-44.25)$ & $23(16.75-32)$ & $47.5(39-64.5)$ & 0.000 \\
\hline Albumin (g/dl), median (IQR) & $3.95(3.67-4.32)$ & $4(3.8-4.5)$ & $3.75(3.4-4.17)$ & 0.001 \\
\hline Total bilirubin (mg/dl), median (IQR) & $0.6(0.43-0.77)$ & $0.53(0.41-0.65)$ & $0.7(0.55-0.81)$ & 0.000 \\
\hline INR, median (IQR) & $0.91(0.78-0.91)$ & $0.9(0.73-1)$ & $0.92(0.73-1.1)$ & 0.58 \\
\hline $\mathrm{CRP}(\mathrm{mg} / \mathrm{dl})$, median (IQR) & $6(12-24)$ & $9.5(6-21)$ & $12(10-48)$ & 0.011 \\
\hline $\mathrm{ESR}(\mathrm{mm})$, median (IQR) & $46(20-83.5)$ & $39(17.25-70)$ & $65.5(27.25-96.5)$ & 0.015 \\
\hline Ferritin (ng/ml), median (IQR), & $187(85-326)$ & $121(72.75-282)$ & $300(165-365)$ & 0.000 \\
\hline D-dimer (ng/ml), median (IQR) & $348(150-562)$ & $275(150-501)$ & $425(168-700)$ & 0.03 \\
\hline Glucose (mg/dl), median (IQR) & $134.9(98.75-178.2)$ & $115(95-157)$ & $156(105-195)$ & 0.007 \\
\hline Creatinine $(\mathrm{mg} / \mathrm{dl})$, median (IQR) & $1(0.8-1.3)$ & $1(0.8-1.3)$ & $1(0.72-1.3)$ & 0.37 \\
\hline Urea (mg/dl), median (IQR) & $29(21-42)$ & $30.5(21.75-41.55)$ & $29(20.25-42.75)$ & 0.45 \\
\hline
\end{tabular}

levels might be potential risk factors for LFT abnormalities (Table 4).

\section{Discussion}

In the current study, we aimed to study liver function test abnormalities in COVID-19 patients and factors affecting them and to evaluate whether LFT abnormalities are related to the severity of COVID-19. In our study, we found that COVID-19 infected patients with LFT abnormalities were older than those with normal LFTs. Similar findings were reported by Cichoż-Lach et al. [5] and Cai et al. [7]. Also, the temperature was higher in patients with LFT abnormalities than those with normal LFTs. These results were similar to the findings of Fan et al. [11]. Like Cai et al. [7], we found that the proportion of diabetic cases was significantly higher in patients with LFT abnormalities than those with normal LFTs. ACE2 expression is substantially increased in diabetic patients treated with ACE inhibitors and angiotensin II type I receptor blockers. It was therefore speculated that upregulation of ACE2 in various organs, such as the lungs and liver, may not only promote SARS-CoV-2 infection, but also raises the risk of developing the extreme and fatal disease [12].

In the current study, at the time of admission, we found that $44 \%$ (52 out of 118 ) of COVID-19 infect-
Table 4. Multivariate logistic analysis of SARS-CoV-2 patients to evaluate factors that might be risk factors for liver function test abnormalities

\begin{tabular}{lcc}
\hline Indicators & OR (95\% Cl) & $P$-value \\
\hline Hemoglobin level & $1.304(1.038-1.638)$ & 0.02 \\
\hline Ferritin level & $1.005(1.001-1.010)$ & 0.01 \\
\hline
\end{tabular}

$O R$ - odds ratio, $\mathrm{Cl}$ - confidence interval

ed patients had LFT abnormalities. Similarly, previous studies showed that LFT abnormalities ranged from $37 \%$ to $69 \%[7,11,13,14]$. Moreover, the percentage of LFT abnormalities reached $80.5 \%$ in patients who died due to SARS-CoV-2 infection [15]. We noted that elevation of transaminases (ALT, AST) (a marker of hepatocyte inflammation) was more common than increased bilirubin level (a marker of cholangiocyte damage). We found that $39.8 \%$ (47 out of 118) had elevated liver enzymes. The percentages of ALT and AST elevation were equal (32\%). In line with our results, several previous studies demonstrated that the percentage of ALT elevation ranged from $18.2 \%$ to $31.6 \%[7,11,14,16]$ and the percentage of AST elevation ranged from $14.8 \%$ to $35.4 \%[7,11,16]$. Only one patient had elevated total bilirubin $(0.8 \%)$. Higher percentages of total bilirubin elevation (5.1\% to $11.5 \%$ ) were reported in several previous studies $[7,11,16]$.

As regards the synthetic function of the liver, the authors also found that $11.8 \%$ (14 out of 118 ) of pa- 
tients had a reduction in serum albumin less than the normal range $(3.5 \mathrm{mg} / \mathrm{dl})$. A higher percentage of albumin reduction (62.8\% of 355 patients) was reported by Fu et al. [17]. Elevation of ALT level and reduction in serum albumin were considered as indices of high mortality in SARS-CoV2 positive patients. In the current study, 7.6\% (9 out of 118) had elevated INR. Wang and his colleagues stated that $58 \%$ of 139 COVID- 19 patients had a prolonged prothrombin time [18].

We found that $2.5 \%$ (3 out of 118) of patients had an acute liver injury. Moreover, multiple previous studies revealed that the percentage of ALI varied from $5 \%$ to $28 \%[7,13,17]$. This wide range of difference in percentage of ALI might be due to differences in the percentage of patients with severe COVID-19 or differences in the percentage of patients with underlying chronic liver diseases.

In our study, the elevations in transaminases (ALT, AST), bilirubin, and INR, and reduction in serum albumin were usually mild. These findings were in line with several recent studies $[5,7,11,25,29]$. In the present study, we found that the highest values for AST and ALT levels were $127 \mathrm{IU} / \mathrm{l}$ and $258 \mathrm{IU} / \mathrm{l}$, respectively. However, Chen et al. [29] reported that the highest values for AST and ALT levels were $1445 \mathrm{IU} / \mathrm{l}$ and $7590 \mathrm{IU} / \mathrm{l}$, respectively.

Although the exact mechanism of LFT abnormalities remains unclear, extreme possible theories have been hypothesized. LFT abnormalities may be due to the direct effect of the virus. Also, Feng et al. [19] supposed that the high viral load of SARS-CoV-2 might play a role in hepatic dysfunction. SARS-CoV-2 enters the hepatocytes and cholangiocytes by binding to ACE2 receptors on the surface of these cells [5]. Higher levels of ACE2 receptors were expressed on the surface of the cholangiocytes. It is understood that the cholangiocyte epithelial cells play a major role in initiating and regulating the immune system response and hepatic regeneration. As SARS-CoV-2 binds to the cell by attachment to ACE2, it was expected that cholangiocyte damage would be more common, because the latter expresses more ACE2 on its surface [20]. However, the normal level of ALP levels and the increased AST/ALT levels do not support the hypothesis of liver injury [12].

Furthermore, immune dysfunction in various forms occurs in COVID-19. So, interleukins and other mediators of inflammation released during SARS$\mathrm{CoV}-2$ infection can initiate hepatocellular injury [12, 21]. Liver function test abnormalities may be druginduced; however, mild LFT abnormalities are reported at baseline in many SARS-CoV-2 patients before significant medication use [21, 22].
It may also be expected that the coagulation dysfunction can cause liver damage due to thrombosis among COVID-19 patients [23]. Also, hepatic hypoxia due to SARS-CoV-2 induced respiratory failure cannot be excluded [6]. However, the pattern of aminotransferase elevation does not support the ischemic hepatitis theory [22]. We should not forget that elevation of aminotransferases may not only be due to hepatic damage but also myositis may play a role. This is supported by increased levels of creatinine kinase, lactate dehydrogenase, and myoglobin that were reported in patients with severe COVID-19 [22, 24]. Clinicians will have to determine whether liver injury is related to an underlying liver disease, drugs used for the treatment of COVID-19, direct effect of the virus, or a complicated disease course. Recent studies proposed several theories on potential mechanisms of liver injury in these patients. This review summarizes current evidence related to hepatobiliary complications in COVID-19 [22, 24].

We observed that LFT abnormalities including AST, total bilirubin, and INR were significantly higher among patients with severe SARS-CoV-2 than those with non-severe infection. Our results were comparable with the meta-analyses of Parohan et al. [20], Wong et al. [25], and Mantovani et al. [21]. These findings can be explained by the high levels of proinflammatory cytokines accompanied by the exaggerated immune response in patients with severe COVID-19 [5, 22].

In the current study, serum albumin level was significantly lower in patients with the severe disease than patients with non-severe disease. Our result was in line with the findings in multiple previous studies $[5,20$, 26-28]. Moreover, reduced serum albumin is established as an independent indicator of severe COVID-19 infection, poor prognosis, and increased mortality [5].

In this study, we found that the levels of hemoglobin, CRP, ESR, serum ferritin, D-dimer, and serum glucose were significantly higher in SARS-CoV-2 patients with LFT abnormalities than those with normal LFTs. Chen et al. [23] also reported a correlation between coagulopathy including raised D-dimer and elevated transaminases in SARS-CoV-2 positive patients. Fan et al. [11] and Xie et al. [16] reported a higher level of CRP among patients with LFT abnormalities than those with normal LFTs.

By analysis of the potential risk factors for LFT abnormalities in our patients, we found that increased hemoglobin levels and high serum ferritin levels might be potential risk factors for LFT abnormalities. Also, Bangash et al. documented that liver dysfunction is associated with high ferritin levels [22]. Serum ferritin is not only a marker of iron storage but also a strong inflammatory marker [30]. So, we can confidently infer 
that the inflammatory cytokine storm plays an important role in LFT abnormalities.

\section{Limitations}

Our study has certain limitations. The first is the retrospective collection of data. Secondly, the data about medication used before admission had not been reviewed. Thirdly, we did not follow up patients during admission to detect the course and effect of liver function test abnormalities. Fourthly, even though we tried to remove the effect of chronic liver disease by excluding chronic viral hepatitis and alcoholic hepatitis, the effect of non-alcoholic fatty liver disease could still not be eliminated. The diagnosis of fatty liver disease involves liver radiology analysis, which could not be commonly done when the medical services during the epidemic were minimal.

\section{Conclusions}

At the time of hospital admission, LFT abnormalities are very common in SARS-CoV-2 positive patients, reaching $44 \%$. Levels of inflammatory response markers including ESR, CRP, serum ferritin, and D-dimer were higher in COVID-19 patients with LFT abnormalities than those with normal LFTs. High serum ferritin levels might be potential risk factors for LFT abnormalities. So, we can confidently infer that the inflammatory cytokine storm plays an important role in LFT abnormalities.

\section{Recommendation}

Monitoring liver function tests is highly recommended, especially for elderly patients and patients with severe SARS-CoV-2 infection. Further studies will be needed to explain the mechanism of liver function test abnormalities.

\section{Acknowledgments}

We would like to thank all health care workers who help us in the collection of data.

\section{Disclosure}

The authors declare no conflict of interest.

\section{References}

1. Mohammed M, Shafik NS, Maher A, et al. Liver injuries in COVID-19 infected patients. Sohag Medical Journal 2020; 24: 15-19.
2. Lai CC, Shih TP, Ko WC, et al. Severe acute respiratory syndrome coronavirus 2 (SARS-CoV-2) and coronavirus disease-2019 (COVID-19): The epidemic and the challenges. Int J Antimicrob Agents 2020; 55: 105924.

3. MacIntosch K. Coronavirus disease 2019 (COVID-19): Epidemiology, virology, and prevention - UpToDate. UpToDate 2021. https://www.uptodate.com/contents/coronavirus-disease-2019covid-19-epidemiology-virology-and-prevention/print.

4. WHO Coronavirus (COVID-19) Dashboard. WHO Coronavirus Disease (COVID-19). https://covid19.who.int/ (accessed March 6, 2021).

5. Cichoż-Lach H, Michalak A. Liver injury in the era of COVID-19. World J Gastroenterol 2021; 27: 377-390.

6. Lei HY, Ding YH, Nie K, et al. Potential effects of SARS-CoV-2 on the gastrointestinal tract and liver. Biomed Pharmacother 2021; 133: 111064 .

7. Cai Q, Huang D, Yu H, et al. COVID-19: Abnormal liver function tests. J Hepatol 2020; 73: 566-574.

8. Zhang C, Shi L, Wang FS. Liver injury in COVID-19: management and challenges. Lancet Gastroenterol Hepatol 2020; 5: 428-430.

9. Li J, Fan JG. Characteristics and mechanism of liver injury in 2019 coronavirus disease. J Clin Transl Hepatol 2020; 8: 13-17.

10. Masoud HH, Zaky S, Baki AA. Management Protocol for COVID-19 Patients Version 1.4/30th May 2020 Ministry of health and population (MOHP), Egypt. 2020.

11. Fan Z, Chen L, Li J, et al. Clinical features of COVID-19-related liver functional abnormality. Clin Gastroenterol Hepatol 2020; 18: 1561-1566.

12. Anirvan P, Bharali P, Gogoi M, et al. Liver injury in COVID-19: The hepatic aspect of the respiratory syndrome - what we know so far. World J Hepatol 2020; 12: 1182-1197.

13. Bloom PP, Meyerowitz EA, Reinus Z, et al. Liver biochemistries in hospitalized patients with COVID-19. Hepatology 2021; 73: 890-900.

14. Li L, Li S, Xu M, et al. Risk factors related to hepatic injury in patients with corona virus disease 2019. medRxiv 2020.

15. Zhang B, Zhou X, Qiu Y, et al. Clinical characteristics of 82 death cases with COVID-19. medRxiv 2020.

16. Xie H, Zhao J, Lian N, et al. Clinical characteristics of non-ICU hospitalized patients with coronavirus disease 2019 and liver injury: a retrospective study. Liver Int 2020; 40: 1321-1326.

17. Fu L, Fei J, Xu S, et al. Acute liver injury and its association with death risk of patients with COVID-19: A hospital-based prospective case-cohort study. medRxiv 2020; 86 .

18. Wang D, Hu B, Hu C, et al. Clinical characteristics of 138 hospitalized patients with 2019 novel coronavirus-infected pneumonia in Wuhan, China. JAMA 2020; 323: 1061-1069.

19. Feng G, Zheng KI, Yan QQ, et al. Covid-19 and liver dysfunction: Current insights and emergent therapeutic strategies. J Clin Transl Hepatol 2020; 8: 18-24.

20. Parohan M, Yaghoubi S, Seraji A. Liver injury is associated with severe coronavirus disease 2019 (COVID-19) infection: a systematic review and meta-analysis of retrospective studies. Hepatol Res 2020; 50: 924-935.

21. Mantovani A, Beatrice G, Dalbeni A. Coronavirus disease 2019 and prevalence of chronic liver disease: A meta-analysis. Liver Int 2020; 40: 1316-1320.

22. Bangash MN, Patel J, Parekh D. COVID-19 and the liver: little cause for concern. Lancet Gastroenterol Hepatol 2020; 5: 529530.

23. Chen S, Liu H, Li T, et al. Correlation analysis of coagulation dysfunction and liver damage in patients with novel corona- 
virus pneumonia: a single-center, retrospective, observational study. Ups J Med Sci 2020; 125: 293-296.

24. Alqahtani SA, Schattenberg JM. Liver injury in COVID-19: The current evidence. United European Gastroenterol J 2020; 8: 509-519.

25. Wong YJ, Tan M, Zheng Q, et al. A systematic review and metaanalysis of the COVID-19 associated liver injury. Ann Hepatol 2020; 19: 627-634.

26. Zhou C, Huang Z, Tan W, et al. Predictive factors of severe coronavirus disease 2019 in previously healthy young adults: a single-center, retrospective study. Respir Res 2020; 21: 157.

27. Wan S, Xiang Y, Fang W, et al. Clinical features and treatment of COVID-19 patients in northeast Chongqinq. J Med Virol 2020; 92: 797-806.

28. Huang C, Wang Y, Li X, et al. Clinical features of patients infected with 2019 novel coronavirus in Wuhan, China. Lancet 2020; 395: 497-506.

29. Chen N, Zhou M, Dong X, et al. Epidemiological and clinical characteristics of 99 cases of 2019 novel coronavirus pneumonia in Wuhan, China: a descriptive study. Lancet 2020; 395: 507-513.

30. Kell DB, Pretorius E. Serum ferritin is an important inflammatory disease marker, as it is mainly a leakage product from damaged cells. Metallomics 2014; 6: 748-773. 